\title{
Mix-Method Research in Applied Psychology ${ }^{1}$
}

\author{
Michela Cortini \\ Università G. d'Annnunzio of Chieti-Pescara (Italy) \\ Email: cortini@unich.it
}

\section{Doi:10.5901/mjss.2014.v5n23p1900}

\begin{abstract}
This paper presents a critical perspective on mix-method research in applied psychology. The starting point is a review concerning the recent development of both quantitative and qualitative paradigms, in the effort of showing how their respective limits may be seen as overlapping. A detailed historical review of the first mix-method approaches is offered, along with a critical presentation of the Methodological Appropriateness Paradigm of Patton. The paper concludes with a brief consideration concerning the problem of synthesis in doing mix-method research.
\end{abstract}

Keywords: mix-method research; triangulation; applied psychology

\section{Introduzione}

Il presente paper intende offrire una riflessione critica sulla querelle tanto invocata nelle scienze sociali, compresa la psicologia, tra metodi quantitativi e metodi qualitativi, proponendo al lettore le ragioni della percorribilità di una via metodologica alternativa: la triangolazione (Fielding \& Fielding, 1986).

Quando si parla di triangolazione si fa riferimento all'incrocio tra punti di vista, siano questi rappresentati da diversi ricercatori e scienziati sociali, e dunque ben venga l'interdisciplinarietà, o siano questi i punti di vista di tecniche di raccolta ed analisi dati distinti.

Proporre la triangolazione di tecniche e metodi di analisi significa, in certo senso, proporre una terza via, tanto poco percorsa per quanto ancora poco apprezzata. Se, infatti, i termini della contesa sono, esplicitamente ed a più riprese, ritratti nei termini di tecniche di raccolta ed analisi dati o quantitativi o qualitativi, è facile intuire che l'integrazione tra i due contendenti sia non solo poco percorsa ma addirittura non legittimata, inconcepibile dal punto di vista della ideologia metodologica. II tutto con la grave conseguenza che tentare una triangolazione rischia spesso di tradursi in una strana posizione per cui non si è riconosciuti né dalla compagine dei ricercatori che si definiscono quantitativi né tanto meno da quella composta dai ricercatori che si definiscono qualitativi e questo perché le due posizioni si sono radicalizzate oltremodo.

Per comprendere appieno non solo la legittimità della posizione triangolare ma la sua piena percorribilità sarà utile fare riferimento ai limiti che metodi quantitativi e metodi qualitativi hanno dimostrato nel tempo.

Potremmo, infatti, sostenere l'ipotesi secondo cui i metodi triangolari si son fatti spazio al di sopra ed al di là della querelle metodi quantitativi vs metodi qualitativi proprio a causa dei limiti che i due noti e maggiori paradigmi hanno rivelato nel tempo.

Nei paragrafi che seguono illustreremo brevemente quelli che a nostro parere sono i limiti maggiori ed i rischi più pericolosi cui si cono spinti i metodi quantitativi e qualitativi nella rigida difesa del proprio quid distintivo rispetto alla controparte.

\section{La Deriva dei Metodi Quantitativi nelle Scienze Sociali}

I metodi quantitativi, incarnati nel paradigma scientista o modernista, il cui indubbio merito, specie con la nascita della psicologia scientifica di stampo wundtiano a cavallo tra '800 e ' 900 , è stato quello di favorire il superamento del metodo filosofico principe, l'introspezione, dando inizio ad una speculazione basata sull'osservazione della realtà, hanno avuto lungo il secolo scorso uno sviluppo incredibile, inscindibilmente legato allo sviluppo che l'informatica ha generato nelle

1 Una prima versione del presente paper compare come Cortini, Michela (2010) La triangolazione dei metodi nella ricerca educativa, in C. Laneve (a cura di) Ci sono dei Posti Vuoti in Classe. Analisi della dispersione scolastica e linee di intervento. Bari, Centro Pedagogico Meridionale. 
scienze computazionali, in primis nella statistica.

Questo sviluppo, di per sé positivo, si è tradotto nel rischio di ridurre le scienze sociali o meglio il lavoro del ricercatore sociale al contare e, sempre di più, al saper maneggiare al meglio tecniche e software statistici. In particolare, lo sviluppo di alcune tecniche statistiche discrezionali, tra le quali va citata in primis l'analisi fattoriale, ha lasciato qualche dubbio circa la possibilità, non per nulla remota a dire il vero, di poter validare sofisticate teorie grazie a tecniche ed aggiustamenti di calcolo statistico, in ultima analisi andando a far pesare, più dello stesso dato empirico, una capacità che si avvicina a quella di "far tornare i conti".

In aggiunta, come ben evidenziato dai teorici delle misure non reattive già sul finire degli anni ' 60 , solitamente i metodi di analisi quantitativi si basano su tecniche di raccolta dati dove è il ricercatore stesso ad imporre il ventaglio di risposte tra le quali i soggetti in indagine sceglieranno (si pensi al metodo della survey), con non poche conseguenze in termini di rischio di manipolazione del setting. In tal senso, il rischio maggiore è quello di validare ottime teorie con sofisticate tecniche statistiche; teorie però che risultano vivide nella mente del ricercatore ma che spesso non hanno un reale riscontro nella realtà in termini di costrutti sotto indagine.

\section{La Rivalutazione dei Metodi Qualitativi nelle Scienze Sociali}

La deriva quantitativa e modernista è stata fortemente messa in discussione a partire dagli ani ' 60 con lo sviluppo delle scienze della comunicazione (si pensi in primis alla teoria degli Atti Linguistici ma anche e soprattutto alla Pragmatica della Comunicazione Umana espressa dalla Scuola di Palo Alto) e con la nascita dell'etnometodologia (Garfinkel, 1967) e dell'interazionismo simbolico (Mead, 1934).

I ricercatori sociali cominciano ad essere insoddisfatti di una scienza a tutti gli effetti computazionale e si interrogano sulla possibilità di adottare come metodo scientifico gli stessi metodi che le persone qualunque usano per dare significato agli eventi in cui sono coinvolti (proposta etnometodologica). La rivoluzione non è da poco; ad un metodo freddo, oggettivo, calcolabile, si sostituisce un metodo soggettivo, "caldo", emico, che vale dal di dentro della stessa realtà sociale. È questa la nascita del fermento qualitativo che si cristallizzerà in paradigma epistemologico, andando a proprorre, al posto delle leggi dell'approccio nomotetico - quantitaivo, le osservazioni del qui ed ora, deprivate di qualsiasi presunzione di validità ma ricche di una potenza euristica che darà l'impulso allo sviluppo di teorie e metodi di intervento psico-sociale affascinanti perché radicati nei dati stessi e non nelle teste dei riecrcatori e tuttora validi, quali per esempio il modello della vita quotidiana di Goffman (1967) piuttosto che la teoria del doppio legame di Watlawick e collaboratori (1967).

\section{La Deriva dei Metodi Qualitativi nelle Scienze Sociali}

II paradigma qualitativo ha recentemente vissuto, a nostro avviso, una deriva paradossalmente simmetrica a quella di cui hanno patito i metodi quantitativi. Stiamo facendo riferimento alla svolta postmoderna nelle scienze sociali, che si è consumata a partire dalla fine degli anni ' 80 e che, a nostro avviso, rappresenta un'involuzione e non, come da molti sostenuto, un'evoluzione (Weick, 1995), andando ad invertire il gioco di potere tra realtà ed interpretazione. In altre parole, il pensiero postmoderno, che nasce dalle ceneri della deriva quantitativa, ha finito col prediligere un approccio che mina l'ontologia stessa dei fenomeni e dei soggetti in indagine a favore dell'interpretante, a favore, nuovamente, del soggettivo. La realtà nella sua oggettività ontologica non sembra non solo interessare ma nemmeno sussistere fintanto che non incrocia un utente di qualche tipo capace di restituirle significato.

Quello che ci spaventa, in altre parole, è il relativismo e la debolezza di una posizione scientifica che afferma ingenuamente (?) che l'unica forza capace di dare vita e sussistenza a cose e fenomeni sia il punto di vista di un soggetto, la sua capacità di dare significato. II dare significato, dovrebbe, invece, a rigor di logica, seguire il dato dell'esistenza.

Superfluo sottolineare come e quanto alle due derive, quella del paradigma quantitativo e quella a seguire del paradigma qualitativo, si siano susseguite posizioni di taglio ideologico sempre più marcato e barricamenti integralisti che hanno portato di fatto a quella inconciliabilità tra metodi e tecniche di raccolta ed analisi dei dati nelle scienze sociali cui abbiamo già fatto riferimento.

\section{La Rivoluzione del Paradigma della Methodological Appropriateness}

La prima interessante proposta di integrazione fra metodi che non rispondesse ai canoni della competizione tra gli stessi risale forse ad un articolo di Wilson, capace di anticipare i tempi e di parlare in questi termini 
"qualitative and quantitative approaches are complementary rather than competitive methods (...and the) use of a particolar method (...) rather must be based on the nature of the actual problem at hand" $(1981$, p.58).

Nel 1990, tale concetto verrà ripreso e finemente articolato da Patton con la sua proposta conosciuta con il nome di Methodological Appropriateness. Questo affascinante binomio sottolinea come il suggerimento per una tecnica di analisi a svantaggio di un'altra debba essere imposto non dai limiti conoscitivi di un ricercatore (in realtà è triste constatare come e quanto ad ogni ricercatore corrisponda una ed una sola, o al massimo due, tecniche di analisi, nella piena ignoranza di altre tecniche e metodiche) ma piuttosto dalla natura dell'oggetto in indagine. Ci sono oggetti di indagine psicosociale, quali per esempio gli effetti psicosociali di eventi drammatici o catastrofici, che non solo non è possibile indagare con una manipolazione sperimentale stretta ma che sarebbe addirittura non etico indagare con un impianto sperimentale.

Con questo non vogliamo certo farci alfieri o proponenti di una figura ricercatore tuttologo in termini di tecniche e metodiche di analisi, ma piuttosto suggerire che le competenze metodologiche specifiche del ricercatore non dovrebbero limitare il ventaglio di scelta tra metodi e tecniche di raccolta ed analisi dei dati.

Un piano di idealità ci sembra quello per cui sia l'appropriatezza metodologica tra natura dell'oggetto in indagine e tecnica di analisi a suggerire una simile scelta; certo non sempre ci riscopriremo come portatori di quelle stesse competenze metodologiche che l'oggetto in indagine ci suggerisce di adottare... ma allora ben venga la triangolazione meno perpetrata nella storia delle scienze sociali: quella tra ricercatori diversi e tra scuole di ricerca diverse.

\section{La Querelle Metodi Quantitativi vs Metodi Qualitativi Tra Scienze di Base e Scienze Applicate}

Nell'esposizione della querelle metodi quantitativi vs metodi qualitativi si fa purtroppo poco spesso riferimento ad una distinzione portante: quella tra scienze pure, o di base, e scienze applicate. Per non andare troppo lontano e per parlare piuttosto di psicologia del lavoro e delle organizzazioni, potremmo fare riferimento da un lato al rigore metodologico che tradizionalmente compete e caratterizza chi si occupa di memoria ed apprendimento e, dall'altro, al pragmatismo metodologico di chi deve suggerire una riforma nel campo giuslavoristico o un intervento a sostegno della riabilitazione di lavoratori che hanno sofferto di stress lavoro-corelato.

La scienza applicata deve rispondere a problemi molto concreti e non al solo impeto conoscitivo, per quanto degno di considerazione e rispettoso quest'ultimo sia.

Detto diversamente, allo scienziato applicato, che si riconosce in un mandato sociale che contempla la capacità di risolvere problemi e di intervenire per sanare una situazione di danno o disagio personale o sociale, è non solo concesso un pluralismo metodologico ma quasi suggerito un eclettismo volto più all'efficacia che non all'accuratezza. Viene alla mente, in tal senso, l'esempio bellissimo, nonostante dia i brividi, riferito da Weick (1995) che racconta come un gruppo di militari ungheresi disperso tra i ghiacci delle Alpi si salvò grazie ad una cartina non solo non accurata ma che addirittura descriveva una realtà geografica distinta, i Pirenei. In tal senso è chiaro come, al limite del paradosso, una tecnica o uno strumento d'analisi del tutto inaccurato possa paradossalmente risolvere problemi anche piuttosto importanti.

Detto con altre parole ancora, richiamando quanto soleva dire Turner, una dei pionieri dell'odierna psicologia sociale, preoccuparsi troppo dei metodi di ricerca scientifica è come darsi da fare incessantemente per preparare le valigie che ci supporteranno durante le agognate vacanze, senza mai partire per il viaggio desiderato.

Può essere utile, in tal senso, richiamare quanto suggerito da Kaplan (1964) in merito alla distinzione tra logica ricostruita e logica in uso; la prima si riferisce alla spiegazione razionale di una metodologia in un settore scientifico dato, esistente in termini ideali; la seconda, viceversa, al modo reale e concreto con cui si fa scienza, dove è naturale scendere a qualche forma di compromesso, venendo meno a quel purismo metodologico che è sostenibile solo in un piano di idealità.

\section{La Triangolazione dei Metodi come Alternativa}

La questione metodologica, intesa come scelta di strumenti e tecnologie con le quali raccogliere ed indagare i dati riferiti ai costrutti teorici oggetto di indagine, in psicologia e, più in generale, nelle scienze sociali, non solo ha marcato l'origine delle nostre discipline in quanto scienze ma le caratterizza, per alcuni addirittura in senso più pesante ed ossessivo riguardo quanto accade alle scienze cosiddette hard (Meehl, 1978).

Questo da un lato non deve stupire o scandalizzare, stante la nostra stessa origine storica, non sempre piana e rassicurante ma piuttosto battagliata nel tentativo di affrancarsi in primis dalla madre comune filosofia; ma dall'altro lato, 
questo stesso insistere sui metodi dovrebbe forse partire da una seria considerazione critica capace di restituire ai metodi quella posizione ancillare, rispetto ai contenuti, che dovrebbero ontologicamente avere. Un metodo eccelso ma mal applicato, o peggio applicato per rispondere a domande stupide 0 a domande che nessuno ha mai posto, è sterile quanto un'ottima speculazione teoretica-filosofica che non abbia nulla da spartire con il piano della realtà. La consapevolezze nelle scelte metodologiche aiuta lo sviluppo e l'avanzamento delle scienze, siano queste naturali o sociali ma forse l'enfasi eccessiva e continua posta alla querelle tra metodi e tecniche quantitativi da un lato e metodi e tecniche qualitative dall'altro rischia di oscurare l'avanzamento delle scienze sociali (Sechrest \& Sidani, 1995).

\section{All'origine Dell'approccio Multi-Metodo}

Nonostante l'enfasi sulla possibilità di triangolare ed integrare tra loro metodi e tecniche diverse sia piuttosto recente, le origini in termini epistemologici datano, ormai, ad una quarantina di anni fa e si radicano in speculazioni, a dire il vero, assai lontane dalle nostre. Più nel dettaglio, fu per primo Smith (1975) ad utilizzare il termine "triangolazione" per significare metaforicamente l'utilizzo sincretico di diverse tecniche di analisi, riprendendo tale termine dal gergo militare dove "triangolare" significa individuare la posizione esatta di un oggetto nello spazio, quale per es. una nave nemica, utilizzando molteplici punti esterni di osservazione tra loro indipendenti.

Se ci allontaniamo dall'uso in senso stretto del termine "triangolazione" e ci interessiamo, piuttosto, dell'idea di integazione in senso lato, possiamo far risalire la riflessione sull'approccio multi-metodo al dibattitto sulle misure non reattive, addirittura a partire dalla fine degli anni ' 50 (Flick, 1992). Intorno a quegli anni, infatti, un gruppo di ricercatori sociali comincia a chiedersi se e a quali condizioni un'ipotesi data regge il confronto con una serie di metodi di verifica tra Ioro complementari (Campell \& Fiske, 1959; Webb et al., 1966; Sechrest \& Phillips, 1979). L'idea è quella di sviluppare una matrice di dati multi tratto - multi metodo, capace di bypassare un bias metodologico che si fa sempre più evidente: il condizionamento, nelle risposte e nelle espressioni dei soggetti in indagine, da parte del metodo utilizzato. In tal senso, potremmo dire, facendo eco a Flick (1992), che la speculazione metodologica rimane comunque all'interno della matrice quantitativo-empirica; non si azzarda una fonte di dati realmente distante, dal punto di vista potremmo dire ontologico, dai metodi e dalle tecniche di raccolta dati quantitativi. Ciononostante, l'intuizione di incrociare tra loro tecniche diverse è sicuramente alla base dell'approccio che oggigiorno chiamiamo triangolare.

Successivamente ed al di là dei teorici delle misure non reattive, va ricordato un altro nome nella storia del costrutto della triangolazione: quello di Denzin che, a partire dagli anni ' 70 , fornisce una speculazione ed una riflessione direi quasi continua sulla possibilità di incrociare metodi e tecniche di raccolta ed analisi dati distinti all'interno del paradigma qualitativo. Potremmo, senza azzardare, sostenere che Denzin ha fatto all'interno del paradigma qualitativointerpretativo, quello che Webb e collaboratori hanno fatto all'interno del dominio quantitativo.

\section{La Triangolazione dei Metodi come Alternativa alla Validazione}

Per cogliere finemente il costrutto di triangolazione, dobbiamo muovere dai primi passi storici evidenziati nel paragrafo precedente ed addentrarci nella speculazione fornita da Denzin; speculazione che ha avuto il merito di generare un vero e proprio fermento intorno all'approccio multi metodo, con non poche critiche al paradigma di Denzin stesso che, nella revisione critica del proprio modello metodologico si è spostato da una certa fissazione sul concetto di validazione ad una più ampia riflessione sulla triangolazione come possibilità generatrice di nuove teorie, come vedremo anche nella riflessione finale sul concetto di sintesi che chiude l'articolo.

Prima di arrivare alla critica della validazione è però forse opportuno inserire il dibattito sulla triangolazione all'interno di quei contributi che hanno segnato il passaggio dalla competizione alla complementarietà tra paradigmi diversi, in primis il paradigma di Patton sulla Methodological Appropriateness (1990) già citato. È questo un passaggio epocale poiché segna un'apertura che i diversi ricercatori aderenti ai due paradigmi contrapposti hanno inteso avviare.

Fino agli anni ' 90 , detto diversamente, sia i ricercatori di stampo quantitativo che i ricercatori di stampo qualitativo hanno sottolineato le differenze di paradigma e rivendicato una sorta di autorità scientifica sul paradigma contrapposto, in virtù della sacralità scientifica delle leggi per quanto concerne l'approccio nomotetico o quantitativo, piuttosto che in virtù di quella che potremmo chiamare la validazione emica, o interna, intesa come alfiere dell'approccio interpretativo 0 qualitativo. Solo con la presa di consapevolezza piena dei limiti dell'approccio proposto, quelle che abbiamo chiamato derive poco sopra, si è passati dal conflitto a viso aperto alla possibilità del dialogo.

Essendo il limite primo dell'approccio nomotetico riconducibile alla fondazione teorica, ed essendo il limite primo dell'approccio qualitativo riconducibile all'impossibilità di una qualche forma di validità esportabile dal qui ed ora dei soggetti indagati alla popolazione cui questi stessi appartengono, si inteso, in una prima fase, proporre dei percorsi 
integrativi fortemente ancorati alle diverse fasi di una ricerca. In tal senso, la manualistica di metodologia della ricerca sociale ha cominciato a suggerire l'utilizzo distinto, e dunque triangolare, di metodi e tecniche qualitative nella fase esplorativa di una ricerca, combinati con metodi e tecniche di natura quantitativa nella fase di validazione. Da un punto di vista logico, tale proposta è interessante, sottolineando come e quanto la sofisticazione metodologica priva di fondamento emico sia poco percorribile (si tratterebbe di validare finemente e con molta, forse eccessiva, accuratezza statistico-matematica, delle teorie che nascono più dalla mente del ricercatore che non dai suggerimenti che la realtà sociale stessa può dare); il concetto di Grounded Theory viceversa, intende riferirsi proprio ad una teoria suggerita dall'analisi di dati grezzi e primi e non dalla speculazione sagace di un ricercatore. Dall'altro lato, il paradigma qualitativo privo di qualsiasi tipo di validità ecologica rimane monco ed incapace di generare una legge scientifica che possa permettere al ricercatore, non solo di base ma anche applicato, di fare previsioni e di intervenire successivamente in maniera adeguata sulla realtà a partire da quelle stesse previsioni; l'unica validità cui può ambire l'approccio qualitativo è confinata nel tempo e nello spazio, con il dubbio corelato che lo sforzo scientifico messo in atto forse non valga la pena.

Se guardiamo però più in dettaglio questa prima proposta di complementarietà, ci accorgeremo, come sottolineato più volte (si veda Flick, 1992 per una rassegna), che dietro l'apparente sincretismo si nasconde una pericolosa visione ipermodernista della scienza, per cui le analisi e le proposte qualitative sembrano, alla fine dei conti, ancillari, alla fase di validazione statistica che, probabilmente anche in virtù di un elevato grado di sofisticazione cui ha conseguito una minore accessibilità e padronanza da parte di molti, si è guadagnata, in termini di rappresentazione sociale su cosa sia scientifico e cosa no, un buon margine di superiorità rispetto al paradigma qualitativo.

La via che i portavoce del paradigma qualitativo hanno inteso adottare per uscire da una simile impasse è quanto meno contradditoria: hanno, infatti, cominciato a riflettere sui correttivi per garantire la validità alla speculazione qualitativa, di fatto riconoscendo la superiorità dei metodi quantitativi e finendo con il scimmiottarli. II primo a cadere in questa trappola è stato Denzin stesso, per il quale la triangolazione di metodi e tecniche all'interno del paradigma qualitativo ha rappresentato la possibilità di una qualche forma di validazione.

"To summarize, methodological triangulation involves a complex process of playing each method off against the other so as to maximize the vailidity of field efforts" (1978, p. 304).

A partire dagli anni '90, Denzin torna sui propri passi (vedi per un commento critico, Flick, 1992) e sottolinea la possibilità d sostituire la validazione con la triangolazione.

Questo passo, a nostro avviso, è stato di sicuro facilitato dalla sempre più nitida constatazione che i problemi e le sfide cui il ricercatore sociale è posto innanzi quotidianamente facciano riferimento sempre di più ad un piano interdisciplinare e sempre meno ad un piano monodisciplinare.

Venendo in tal senso al dominio specifico della psicologia del lavoro e delle organizzazioni, potremmo in primis sottolineare la natura liminare della suddetta scienza, che si propone, sia in termini di ricerca di base che, a maggior ragione, in termini di scienza applicata, tra psicologia, pedagogia, filosofia e sociologia, e che per questa sua natura richiede una triangolazione di metodi, molto di più e con un'urgenza non paragonabile a quanto accade in domini monodisciplinari.

La sfida maggiore, in questi termini, è come sia possibile effettivamente integrare, e non giustapporre, paradigmi di ricerca ed approcci teorici tra loro distanti, come vedremo nel paragrafo conclusivo.

\section{II "Problema" della Sintesi}

Probabilmente la questione più spinosa, sebbene raramente approcciata nella letteratura sulla triangolazione, riguarda come sintetizzare tra loro dati ed analisi che appaiono spesso contradditori e comunque distanti.

In altre parole, nonostante la triangolazione ed integrazione di metodi e tecniche di analisi diversi sia considerato un punto di arrivo, a noi sembra piuttosto un ottimo punto di partenza, cui, però, deve seguire una fine ed attenta capacità di sintesi che, a dire il vero, non sempre si riscontra, nonostante qualche eccezione (Benevene \& Cortini, 2010; Verrocchio et al., 2012); nei report di ricerca, in altre parole, il più delle volte si trovano giustapposte le discussioni concernenti analisi diverse e manca spesso una sintesi finale. A supporto della sintesi, andrebbe maggiormente esplorata la possibilità offerta da alcuni software, specie per quanto concerne l'analisi testuale, di ispezionare ed analizzare parallelamente lo stesso corpus di dati, mettendo in paragone indici di natura statistico-quantitativa con i dati testuali e discorsivi originali; si pensi per esempio, al software T-Lab (Cortini \& Tria, 2014).

Le caratteristiche che una buona sintesi dovrebbe possedere fanno capo principalmente alla possibilità di far dialogare tra loro risultati di natura diversa, dove l'approccio qualitativo "illumina" le controversie o le opacità cui è giunta 
l'analisi quantitativa e viceversa.

Per concludere sul problema della sintesi, possiamo far riferimento al dibattito che l'impianto metodologico proposto da Denzin ha suscitato a partire dalla fine degli anni ' 80 ; dibattito che ha ridisegnato i contorni ed i confini della querelle metodi qualitativi - metodi quantitativi spostandola da un piano di validazione delle ipotesi, cui, per essere chiari, rimanevano ancorati i teorici delle misure non reattive, ad un piano di generazione di ipotesi e nuove teorie, come abbiamo già evidenziato.

\section{References}

Benevene, P., \& Cortini, M. (2010). Human resource strategic management in NPOs: An explorative study on managers' psychosocial training. Journal of Workplace Learning, 22(8), 508-521.

Campbell, D. \& Fiske, D. (1959). Convergent and discriminant validation by the multi-trait, multi-method matrix, Psychological Bulletin, $56,81-105$.

Cortini, M., \& Tria, S. (2014). Triangulating qualitative and quantitative approaches for the analysis of textual materials: An introduction to T-lab. Social Science Computer Review, 32(4), 561-568.

Fielding, N. G. \& Fielding, J. L. (1986) Linking Data. Beverly Hills, Sage.

Flick, U. (1992) Combining methods. Lack of methodology: discussion of Sotirakopoulou \& Breakwell, Ongoing Production on Social Representations, 1, 1, $43-48$.

Mead, G.H. (1934) Mind, self and society from the standpoint of a social behaviorist. Chiacago, University of Chicago Press.

Meehl, P. E. (1978) Theoretical risks and tabular asterisks: sir Karl, sir Ronald, and the slow progress of soft psychology, Journal of Consulting and Clinical Psychology, 46, 806-834.

Patton M.Q. (1990), Qualitative evaluation and research methods. Newbury Park, Sage.

Sechrest, L. \& Phillips, M. (1979). Unobtrusive measures, an overview. In L. Sechrest (a cura di), Unobtrusive measurement today (pp.117). San Francisco: Jossey Bass.

Sechrest, L. \& Sidani, S. (1995) Quantitative and qualitative methods: is there an alternative?, Evaluation and Program Planning, 18, 1, 77-87.

Smith, H. W. (1975) Strategies for Social Research. Englewood Cliffs, Prentice Hall.

Verrocchio, M. C., Cortini, M., \& Marchetti, D. (2012). Assessing child sexual abuse allegations: An exploratory study on psychological reports. International Journal of Multiple Research Approaches, 6(2), 175-186.

Webb, E., Campbell, D., Scharwtz, R. \& Sechrest, L. (1966) Unobstrusive Measures: Nonreactive Research in the Social Sciences. Chiacago, Rand McNally.

Weick, K. E. (1995) Sensemaking in Organizations. Newbury Park, Sage Publication.

Wilson, T. (1981) Qualitative "versus" quantitative methods in social research, in M. Küchler et al. Integration von qualitativen und quantitativen Forschungsansätzen, Mannheim, ZUMA. 